Article

\title{
Beam Scanning Capabilities of a 3D-Printed Perforated Dielectric Transmitarray
}

\author{
Andrea Massaccesi *(D), Gianluca Dassano and Paola Pirinoli \\ Department of Electronics and Telecommunications, Polytechnic University of Turin, 10129 Turin, Italy; \\ gianluca.dassano@polito.it (G.D.); paola.pirinoli@polito.it (P.P.) \\ * Correspondence: andrea.massaccesi@polito.it; Tel.: +39-0110904209 (ext. 4209)
}

Received: 11 February 2019; Accepted: 23 March 2019; Published: 28 March 2019

\begin{abstract}
In this paper, the design of a beam scanning, 3D-printed dielectric Transmitarray (TA) working in Ka-band is discussed. Thanks to the use of an innovative three-layer dielectric unit-cell that exploits tapered sections to enhance the bandwidth, a $50 \times 50$ elements transmitarray with improved scanning capabilities and wideband behavior has been designed and experimentally validated. The measured radiation performances over a scanning coverage of $\pm 27^{\circ}$ shown a variation of the gain lower than $2.9 \mathrm{~dB}$ and a 1-dB bandwidth in any case higher than $23 \%$. The promising results suggest that the proposed TA technology is a valid alternative to realize a passive multibeam antenna, with the additional advantage that it can be easily manufactured using 3D-printing techniques.
\end{abstract}

Keywords: transmitarray antenna; beam scanning; planar lens; discrete lens; tapered matching; 3D-printed antenna; 3D-printing; additive manufactuing

\section{Introduction}

The next generation of high performing radar and satellite communication systems is requiring the development of enhanced technologies for the design and realization of its components. For what concerns antennas, the main constraints are related to the frequency bands, moving from microwave to millimeter or sub-THz frequencies, the high gain and the multibeam or beam scanning capabilities. To generate wide scanning antennas with good performances in all the pointing directions, the use of active arrays is the most straightforward solution, even if they are characterized by high complexity, that increase dramatically with the number of array elements; moreover, at the higher frequencies, the poor performances of the feeding network represent a limitation to this type of antenna. An alternative is that of using a mechanical beam-steering mechanism, easily implementable in reflector-type antennas. To reduce the antenna volume, recently, solutions where Reflectarray (RA) or Transmitarray (TA) antennas substitute the reflector are taken into account, since they represent a convenient and efficient alternative for obtaining high gain and low cost antennas [1,2]. A transmitarray is typically composed of a feed source that illuminates a single or multilayer quasi-periodic array, whose unit-cells are characterized by one or more variable geometrical parameters, through which it is possible to control the transmission coefficient phase of each cell and to obtain the desired radiation pattern [2]. The TA unit-cell can consist in metallic elements printed on different dielectric layers [3-5], in completely metallic slot-based layers separated by air gaps [6,7] or even in a dielectric structure perforated by one or several holes, as implemented in [8-13]. In comparison with reflectarrays, TAs present the advantage to not suffer for blockage, which require the use of off-set feeds in RA, and this increases the difficulty to implement efficiently the beam scanning.

In view of their features, transmitarrays have been widely considered for the realization of multibeam [14,15] or beam scanning antennas [16-27]. The most straightforward way to obtain the beam steering is that of integrating active elements in the unit-cell, such as p-i-n diodes [16-20] or 
varactor diodes [21-27]: the resulting radiation features are good, but the same considerations already done for active arrays apply. An alternative solution can be the use of a passive TA illuminated by a moving feed or by a feed array, generating beams that impinge on the TA surface with different incident angles and are therefore focused by the TA itself in different directions [14-18]. The main limitation of this solution is its reduced scanning capabilities: in [16], a small size configuration shows a reduction of the gain of approximately $4 \mathrm{~dB}$ on a scanning range from $-30^{\circ}$ to $30^{\circ}$. Some improvements can be obtained using a larger TA, so that just one part of it is illuminated by each incident field $[15,17]$, or designing the TA so that it acts as a bifocal lens [18], even if in this case a degradation of the beam in the broadside direction occurs.

In this paper, the design of the 3D-printable dielectric transmitarray with beam-scanning capabilities is discussed. It adopts the wideband dielectric unit-cell already introduced in [12] are investigated which consists of three perforated layers: the central presents a square hole, which is used to control the phase of the transmission coefficient, while the external layers are equal and have a truncated pyramid hole, which allow for improving the bandwidth and the efficiency. This cell element was firstly introduced in [10-13], which consists of three perforated layers: the central presents a square hole, which is used to control the phase of the transmission coefficient, while the external layers are equal and have a truncated pyramid hole, which allow for improving the bandwidth and the efficiency. This cell element was firstly introduced in [10], where its wideband behavior was tested only with numerical simulations of a medium size TA, designed using a conventional dielectric material and without considering the possible limitations introduced by the manufacturing process. In [11], the enhanced performances of the same unit-cell were compared with those of a conventional unit-cell having just a single layer with a square hole; in this case, a material usable for the realization of the TA with an Additive Manufacturing (AM) technique was considered, since this seemed to be the only adoptable method for the TA fabrication, but also in that work the designed antenna was only numerically analyzed, neglecting the constraints imposed by the AM process. The results in [11] proved that the TA with the tapered unit-cells provided a 1-dB bandwidth doubled (27.6\%) with respect to that of the TA using the single layer cells (13.4\%). The unit-cell was instead experimentally validated in [12]: firstly, several numerical tests were conducted to find the optimum geometric parameters and transmitarray configuration at Ka-band; then, the unit-cell was modified to make it 3D-printable in view of the limitations introduced by AM techniques; finally, a $15.6 \lambda_{0} \times 15.6 \lambda_{0}$ antenna was designed, manufactured with 3D-printing techniques and experimentally characterized in the anechoic chamber. All of the configurations considered in [10-12], were designed neglecting the effect of the direction of arrival of the incident field on the unit-cell and they are only center-fed transmitarrays that generate a main beam in the broadside direction. In [13], some very preliminary considerations on the possibility to realize a mechanically steerable transmitarray are summarized: two different unit-cells are considered, one of which is the dielectric one; small size TAs working at $5.6 \mathrm{GHz}$ were designed without taking into account any manufacturing limitations and considering the possibility to design a bifocal configuration or to use a global optimizer, and then characterized numerically. In this paper, a systematic analysis of the scanning beam capabilities of the unit-cell is first performed and the obtained results are presented in Section 2; the unit-cell has then been used for the design of a medium size TA, considering the steering of the incident field varying between $-30^{\circ}$ and $+30^{\circ}$ : the adopted design procedure is described in detail in Section 3. A prototype has been therefore manufactured and measured: the results collected in Section 4 prove that the TA provides excellent radiation performances for different scanning angles, still maintaining for each of them a large bandwidth behavior; the 1-dB bandwidth remains almost the same for the different pointing angles, which are very stable over the entire bandwidth. Finally, in Section 4, some concluding remarks and possible future actions devoted to further improve the transmitarray features are discussed. 


\section{Unit-Cell Analysis}

The basic structure of the unit-cell (UC) used for the design of the beam scanning TA was described in detail in [12], where it was proposed as an alternative solution to improve the bandwidth in transmitarray antennas; however, for the sake of clearness, its main features are summarized in the following.

As illustrated in Figure 1, the UC consists of three overlapping layers, made of the same dielectric material. The central layer has a square hole, whose size $d$ is varied to control the phase of the transmission coefficient $\left(S_{21}\right)$. The two external elements are characterized by a truncated pyramid hole that connects the hole in the central layer to the external aperture on the unit-cell external top (bottom) side, whose size $W$ is the same for all of the unit-cells. The three layer configuration can be described with the equivalent transmission line model shown in Figure 2. The comparison with the lateral view of the unit-cell in Figure $1 \mathrm{~b}$ highlights that the mid layer behaves as a uniform transmission line with characteristic impedance $Z_{s q h}$, while the external identical layers are equivalent to tapered transmission lines whose characteristic impedance $Z_{\text {tap }}^{*}(z)$ varies linearly along the $z$-axis, and therefore they act as wideband impedance inverters that match $Z_{\text {sqh }}$ to the free-space impedance $\left(Z_{0}\right)$. Both $Z_{s q h}$ and $Z_{s q h}$ are related to the effective permittivity of the corresponding layer that in its turn depends on the relative dielectric constant of the material and the size of the hole. The dimension of the tapered section changes linearly with $z$, and, as a consequence, the characteristic impedance $Z_{\text {tap }}^{*}(z)$ follows the same linear law. As discussed in [10], a good matching can be realized choosing the lengths $l_{1}$ and $l_{3}$ of the tapered lines at least equal to $\lambda_{g} / 2$, where $\lambda_{g}$ is the effective wavelength in the tapered section.

(a)

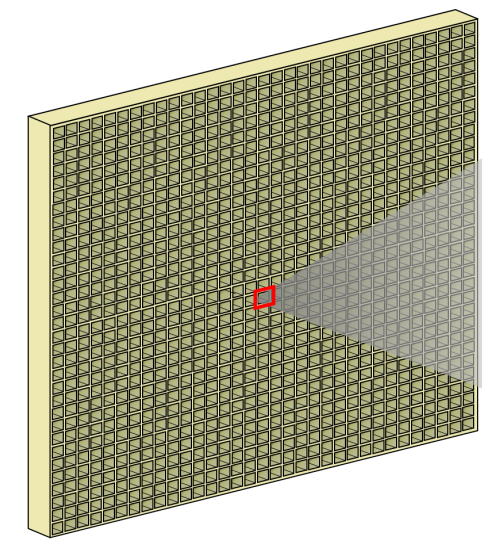

(c)

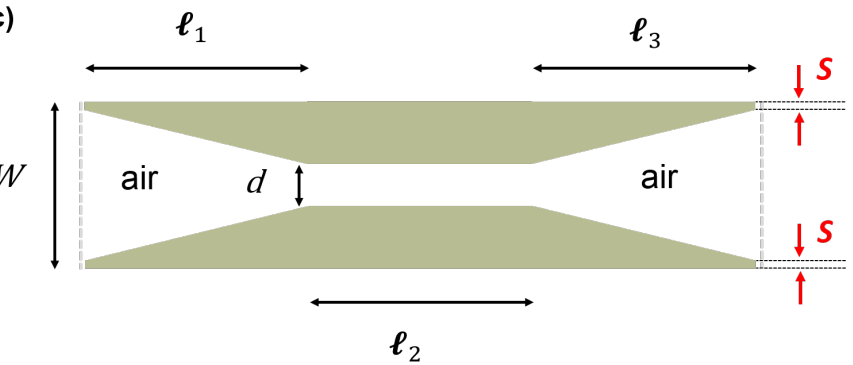

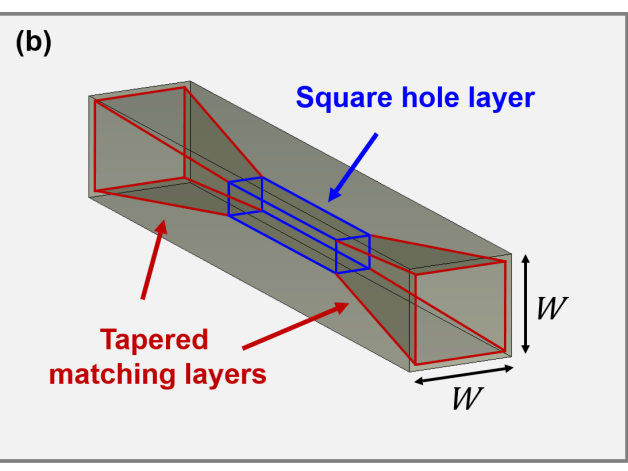

(d)

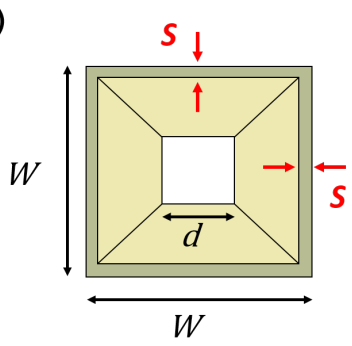

Figure 1. Sketch of a dielectric transmitarray and blow out of the unit-cell structure. (a) dielectric TA; (b) UC perspective view; (c) UC side view; (d) UC top view.

The unit-cell was designed to work in Ka-band, at a frequency $f_{0}=30 \mathrm{GHz}$. To make possible its manufacturing with a 3D printer, it was designed using the commercial dielectric VeroWhite Plus (RGD835 provided by Stratasys ${ }^{\circledR}$ ). This material was electromagnetically characterized using the waveguide method through which it was estimated that the average value of the dielectric constant in the considered frequency range is $\varepsilon_{r}=2.77$ and the loss tangent is $\tan \delta=0.021$. Considering the requirements introduced by the manufacturing process discussed in [12], and the parametric analysis 
performed to determine the optimal sizes of the unit-cell, its side is fixed to $W=0.3 \lambda_{0}=3 \mathrm{~mm}$, while the heights of each layer are $l_{1}=l_{2}=l_{3}=11 \mathrm{~mm}$, corresponding to a total cell thickness $T=l_{1}+l_{2}+l_{3}=3.3 \lambda_{0}=33 \mathrm{~mm}$. Moreover, the resolution of the adopted 3D printer forces the maximum and minimum size of the holes to be equal to 2.65 and $0.5 \mathrm{~mm}$, respectively; this choice does not allow for obtaining a perfect matching between the mid layer equivalent characteristic impedance and $Z_{0}$, with a consequent decrease of the amplitude of $S_{21}$, while a range of $360^{\circ}$ is still achieved for its phase.

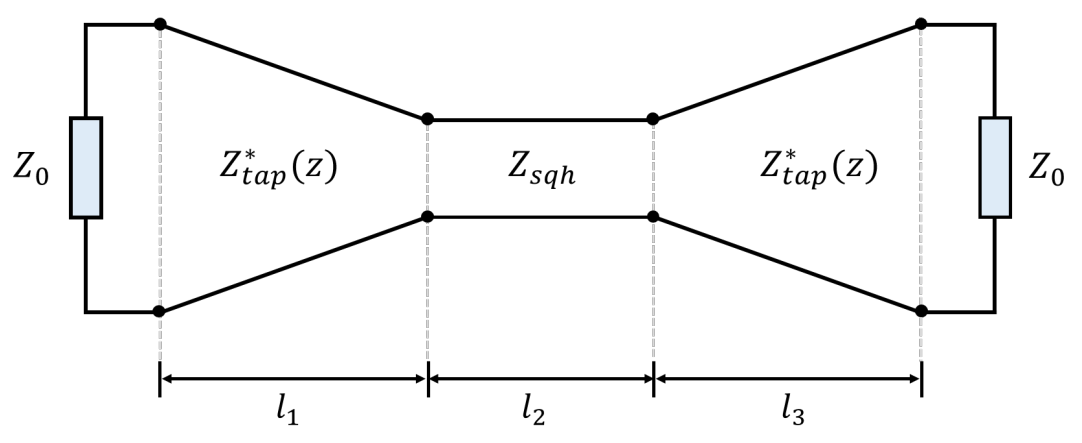

Figure 2. Equivalent transmission line model of the perforated dielectric unit-cell.

As also pointed out in [16], the scanning capabilities of a transmitarray depend, in the first instance, on the feature of the unit-cell, whose behavior must be as less as possible subject to the direction of arrival of the unit-cell, i.e., both the phase and the amplitude of $S_{21}$ have to stay almost the same when the angle of incidence varies. In fact, this feature guarantees that, changing the angle of incidence, no higher modes resonate, as occurs for other unit-cell (see for instance [13]), degrading the antenna radiation performances and also that, when the direction of arrival changes, due to a variation of the position of the feed, the behavior of the unit-cell is not completely different from the presumed one. To study what happens in the unit-cell considered here, it has been analyzed using the Floquet excitations in CST MW Studio, used to compute the transmission coefficient as a function of $d$, for several angles of incidence $\theta_{i}$ (see inset in Figure 3a for its definition). The curves representing the variation of the amplitude and the phase of $S_{21}$ are shown in Figure 3a,b, respectively. They have been calculated at $f_{0}$ and for $\theta_{i}=10^{\circ}, 20^{\circ}, 30^{\circ}, 40^{\circ}$. Since the analyses for TE $\left(\phi_{i}=0^{\circ}\right)$ and TM $\left(\phi_{i}=90^{\circ}\right)$ modes provided the same results, only a single curve is plotted for each incidence angle. As it can be observed in Figure 3a, the amplitude of $S_{21}$ is slightly affected by direction of arrival of the incident wave, with a reduction not greater than $0.5 \mathrm{~dB}$ when $\theta_{i}=40^{\circ}$. For what concerns the phase, from Figure $3 b$, it emerges that both its linearity and full range of variation are not affected by $\theta_{i}$ : in fact, the different curves are almost a translated copy of the other, with small changes in the slope. This means that the dielectric unit-cell behavior is not particularly sensitive to the angle of incidence, and this makes it a potential good candidate for the realization of a beam scanning TA, more suitable than other unit-cells, as some of those using metallic printed elements, where the oblique incident wave excites superior modes, at their turns responsible for a strong reduction in the amplitude of the transmission coefficient and discontinuities in the phase variation. 


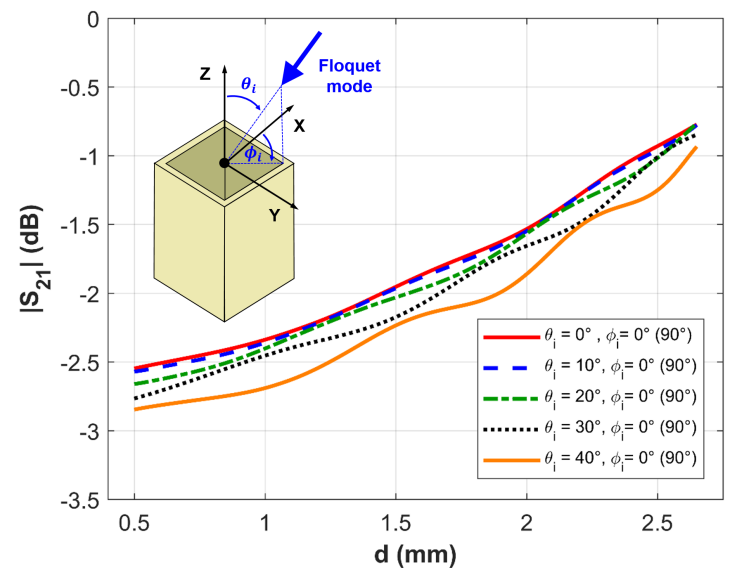

(a)

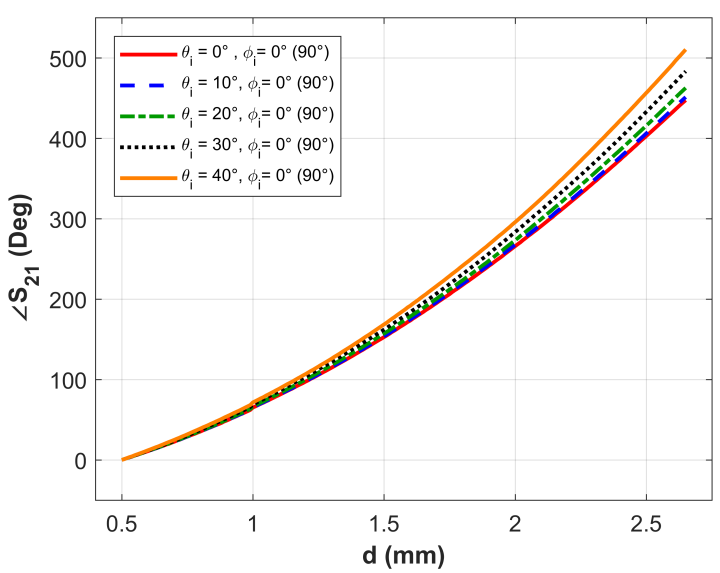

(b)

Figure 3. Comparison of the unit-cell transmission coefficient $S_{21}$ computed at $f_{0}$ as a function of $d$ for several angles of incidence $\theta_{i}$ : (a) amplitude; (b) phase.

\section{Beam Scanning Transmitarray Design}

Adopting the unit-cell described in the previous section, a medium-size transmitarray has been designed: it is composed by a matrix of $50 \times 50$ elements, corresponding to a size $D=150 \mathrm{~mm}=15 \lambda_{0}$ at $f_{0}$. The first step in the design procedure concerns the evaluation of the required phase distribution. The transmission phase of each element is designed to compensate the spatial phase delay from the feed source to it [2]. Considering the coordinate system illustrated in Figure 4, the required phase distribution to obtain a focused beam in a direction normal to the surface is given by:

$$
\psi_{r e q}(m, n)=k_{0}\left(\overrightarrow{\mathbf{r}}-\overrightarrow{\mathbf{r}}_{m n} \cdot \hat{u}_{o}\right)+\psi_{0}
$$

where $k_{0}$ is the propagation constant in free-space, $\overrightarrow{\mathbf{r}}$ represents the distance from the feed to the $(m, n)$-th element, whose position is located by $\overrightarrow{\mathbf{r}}_{m n}$, and $\hat{u}_{0}$ is the unit vector describing the main beam direction. The vector $\overrightarrow{\mathbf{r}}_{f}$ determines the feed position, which is considered to be aligned to the center of the TA surface. The quantity $\psi_{0}$ is a constant phase value, indicating that a relative phase rather than the absolute transmission phase is required for the transmitarray design. Using the array theory, the far-field radiation pattern for a rectangular aperture transmitarray of $M \times N$ elements can be predicted using the following approximated expression:

$$
E(\theta, \phi)=\sum_{m=1}^{M} \sum_{n=1}^{N} \cos ^{q_{e}}(\theta) \frac{\cos ^{q_{f}}\left(\theta_{f}(m, n)\right)}{\left|\overrightarrow{\mathbf{r}}_{m n}-\overrightarrow{\mathbf{r}}_{f}\right|} \cdot e^{-j k\left(\left|\overrightarrow{\mathbf{r}}_{m n}-\overrightarrow{\mathbf{r}}_{f}\right|-\overrightarrow{\mathbf{r}}_{m n} \cdot \hat{u}\right)} \cdot\left|S_{21}(m, n)\right| e^{j \angle S_{21}(m, n)},
$$

where $q_{e}$ is the element power factor, $q_{f}$ is the feed pattern power factor, $\theta_{f}(m, n)$ is the spherical angle in the feed coordinate system in correspondence of each element of the TA and for which the field radiated by the feed is computed. $\left|S_{21}(m, n)\right|$ and $\angle S_{21}(m, n)$ are the magnitude and phase of the transmission coefficient of each element, respectively. Once the required transmission phase $\psi_{\text {req }}(m, n)$ is determined for each element of the TA aperture, the corresponding element dimension is obtained using the curve that relates it to the phase $\angle S_{21}(m, n)$. This curve is generally obtained from the full-wave analysis of the unit-cell, as performed in Section 2. Typically, the evaluation $\angle S_{21}(m, n)$ is made under the assumption that all cells are illuminated by a field with a normal incidence. Actually, most of the elements are illuminated by oblique incidence angles, and therefore this approximation produces a phase error that depends on the degradation of the unit-cell performances with the angle $\theta_{f}$. The incidence angle on each element of the aperture is given by

$$
\theta_{f}(m, n)=\operatorname{acos}\left(\frac{|\mathbf{r}|}{\left|\mathbf{r}_{f}\right|}\right) \text {. }
$$


The value of the incidence angles $\theta_{f}(m, n)$ in correspondence with the TA elements in the case considered here is shown in Figure 5a. It has been computed considering a focal length $F=150 \mathrm{~mm}$ $(F / D=1)$. The angle is maximum at the extremes of the aperture, achieving a peak value of about $34^{\circ}$. The phase error introduced when the normal incidence is assumed for all the unit cells instead of the real direction of arrival of the incidence field is given by

$$
\psi_{\text {error }}(m, n)=\angle S_{21}(m, n)-\angle S_{21}\left(\theta_{f}(m, n)\right),
$$

where $\angle S_{21}(m, n)$ is the transmission phase computed with a normal incidence $\left(\theta_{f}=0^{\circ}\right)$, while $\angle S_{21}\left(\theta_{f}(m, n)\right)$ takes into account the different behavior of the UC with varying $\theta_{f}$ and it has been evaluated from the curves obtained in Figure $3 \mathrm{~b}$. In Figure $5 \mathrm{~b}$, the resulting phase error distribution $\psi_{\text {error }}(m, n)$ for the $50 \times 50$ TA is plotted: as it can be observed, at the center, the phase error is lower than $5^{\circ}$ and it can be considered negligible, while beyond the phase jump, the error increases arriving up to about $30^{\circ}$. It is higher when the position of the feed changes, as occurs during the mechanical beam scanning considered here. For instance, if we consider a scanning angle of $-30^{\circ}$, the distribution of the incident angles becomes that in Figure $5 c$, in which the maximum angle achieved on the right corners is $51^{\circ}$. In Figure $5 \mathrm{~d}$, it can be observed that the phase error related to this case has increased, reaching a maximum of about $45^{\circ}$. Moreover, another important aspect has to be taken into account when the feed is rotating. If the Transmitarray is designed as a center-fed configuration, it is necessary to consider an additional phase error due to the difference between the required phase distribution needed when considering a different feed angle position and the required phase distribution for the center-fed case. Thus, the total phase error for scanning angles is the following:

$$
\left.\psi_{\text {error }}(m, n)\right|_{\text {scan }}=\left[\left.\psi_{\text {req }}(m, n)\right|_{\text {scan }}-\psi_{\text {req }}(m, n)\right]+\psi_{\text {error }}(m, n),
$$

where $\psi_{r e q}(m, n)$ is the required phase distribution for the center-fed case expressed by Equation (1), $\psi_{\text {req }}(m, n)_{\text {scan }}$ is the required phase shift needed for scanning angle case and $\psi_{\text {error }}(m, n)$ is the phase error related to the incidence angles by Equation (4). The second term of Equation (5) can be removed by choosing properly the values of the transmission phase $\angle S_{21}\left(\theta_{f}(m, n)\right)$. This method has been applied to find the optimal phase distribution that allows for reducing the phase error for all the considered scanning angles.

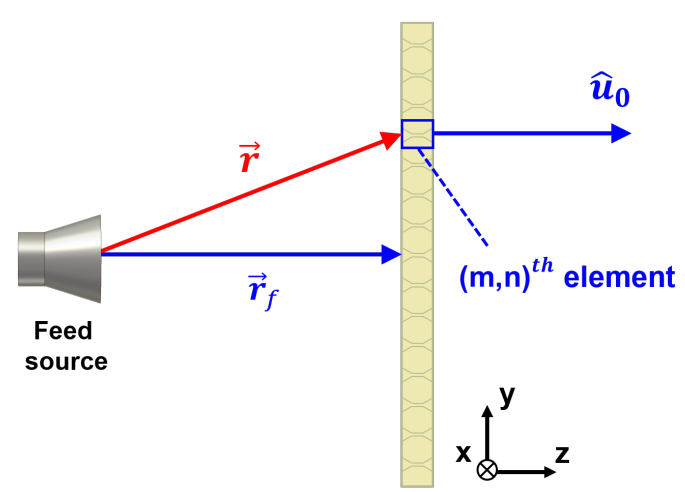

(a)

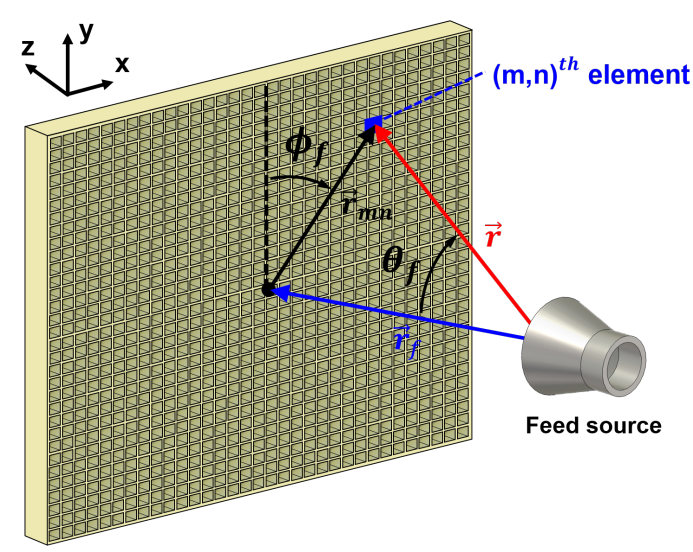

(b)

Figure 4. Sketch of the TA configuration with the adopted coordinate system: (a) side view; (b) perspective view of the illuminated surface.

The feed is a circular horn working in Ka-band: it has a gain of $17 \mathrm{~dB}$ at $30 \mathrm{GHz}$ and its pattern can be modelled as $\cos ^{q_{f}}(\theta)$ with $q_{f}=12.5$. The focal distance between the horn and the dielectric structure is $F=150 \mathrm{~mm}(F / D=1)$. Since here the main interest is in investigating the scanning capabilities of the TA, the beam steering that is obtained with a relative rotation between the two 
parts of the antenna, i.e., the transmitting surface and the feed, and this is equivalent to moving the horn along a circular arc having the center coincident with that of the TA itself, has been sketched in Figure 6a. In this way, the offset angle $\theta_{f}$ of the field impinging the TA changes, and consequently the direction of the main beam, is denoted with $\theta_{b}$. A range of variation for $\theta_{f}$ of $60^{\circ}$ in the E-plane has been considered for the transmitarray proposed here.

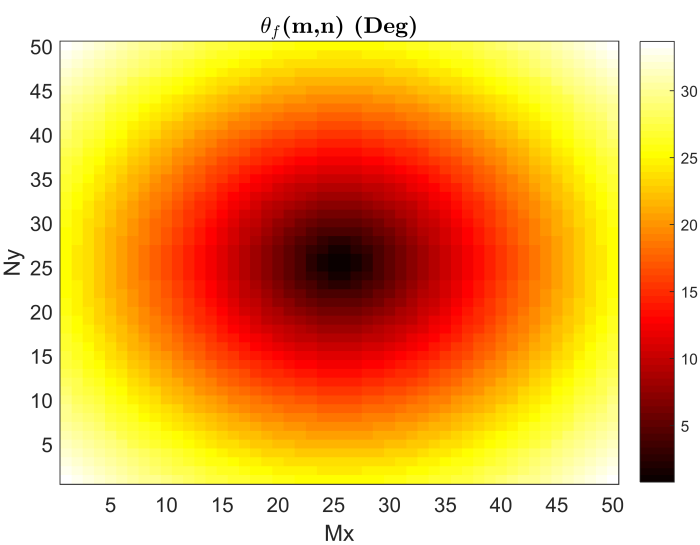

(a)

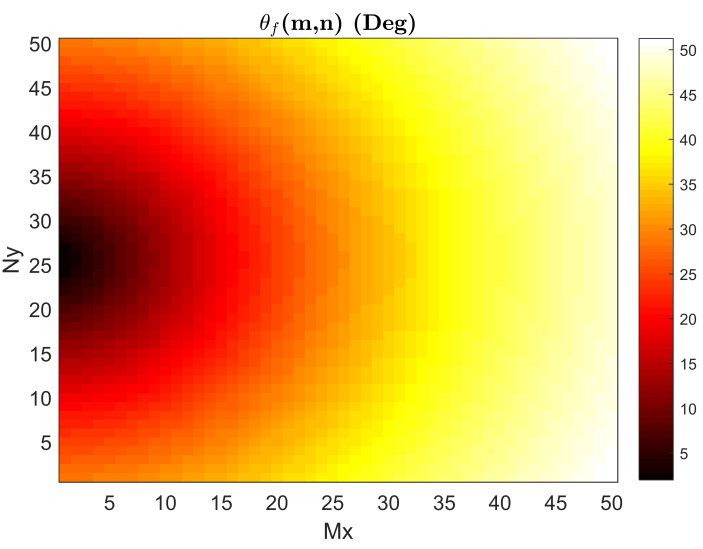

(c)

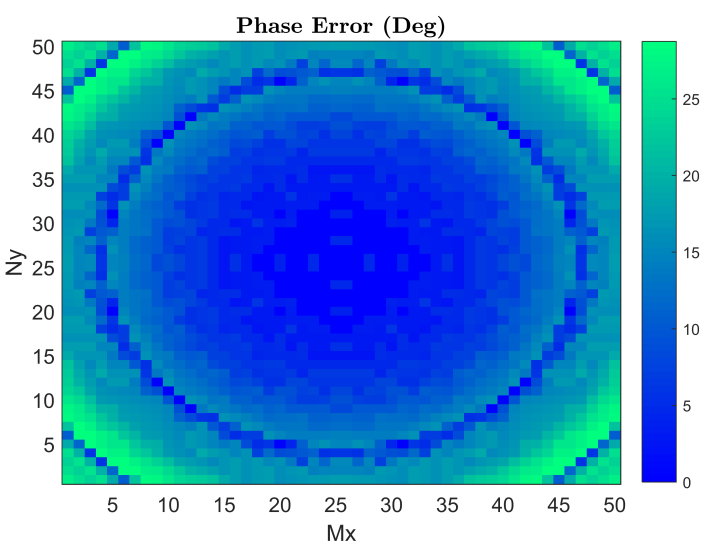

(b)

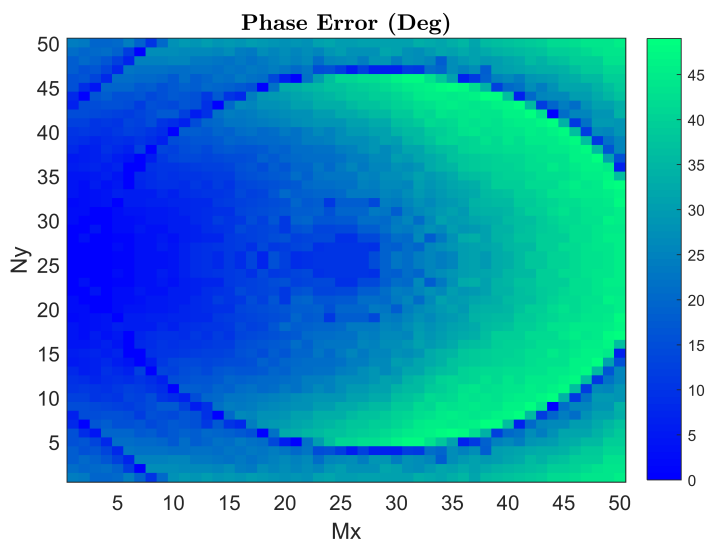

(d)

Figure 5. Matrix of the incidences angles $\theta_{f}(m, n)$ and related phase error: (a) incident angles for a center-fed TA; (b) phase error distribution considering a normal incidence; (c) incident angles when the scanning angle is $-30^{\circ} ;(\mathbf{d})$ phase error distribution considering a normal incidence.

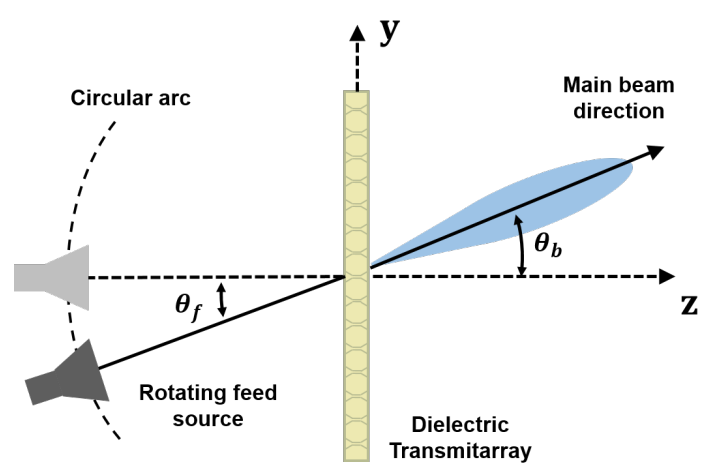

(a)

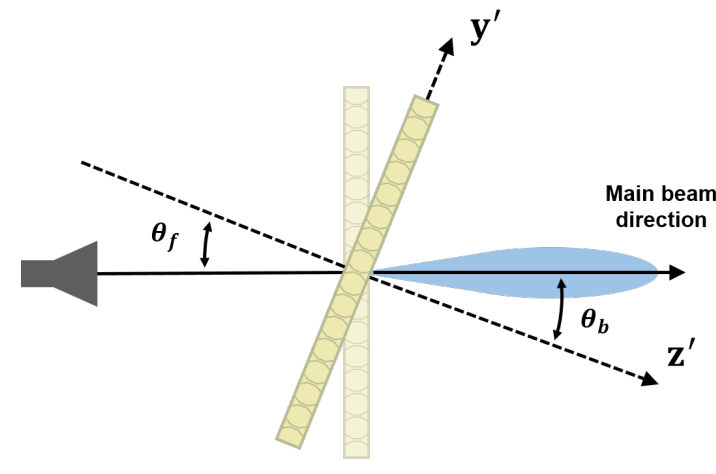

(b)

Figure 6. Pictorial view of the beam scanning mechanism (a) and of its actual implementation (b). 


\section{Prototype Manufacturing and Experimental Characterization}

The performances of the TA have first been numerically analyzed using CST MW Studio; then, a prototype has been manufactured and experimentally characterized in an anechoic chamber. The prototype has been fabricated using the 3D-printer Object30 by Stratasys ${ }^{\circledR}$ that exploits PolyJet technology and allows for achieving a nominal resolution of $100 \mu \mathrm{m}$. In addition, the support connecting the feed to the TA and allowing its movement in the E-plane has been manufactured through a 3D-printing technique and, in particular, an FDM (Fused Deposition Modelling)-based 3D-printer has been exploited to realize it. A photo of the entire antenna and its measurement setup in the anechoic chamber is shown in Figure 7.

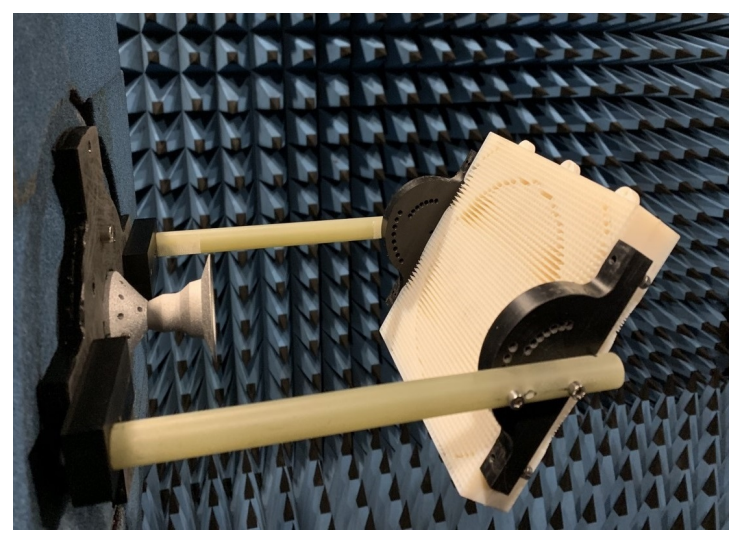

(a)

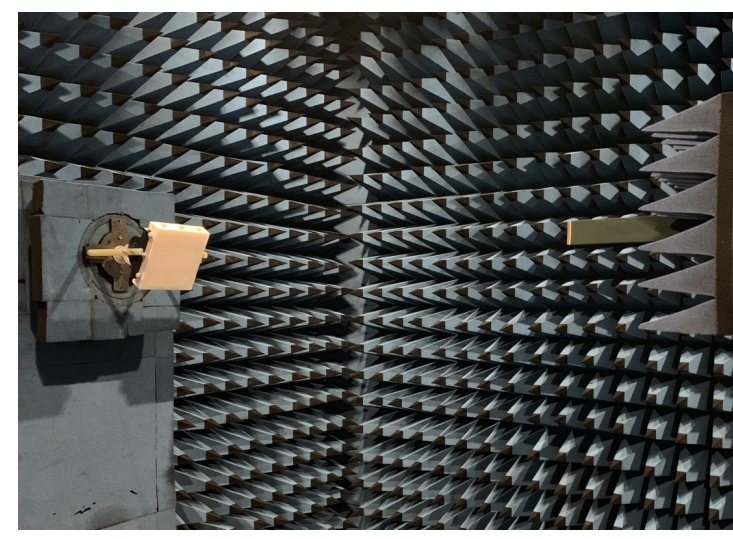

(b)

Figure 7. Beam scanning TA mounted in the anechoic chamber: (a) manufactured prototype and its support structure; (b) measurement setup including the open-ended waveguide probe.

Note that, to simplify the antenna assembly, the beam steering mechanism illustrated in Figure 6a is implemented in a slightly different way, as appears from Figure 7a more clearly from the sketch in Figure 6b: it is the TA that rotates around an axis in the horizontal plane, namely the $x$-axis in the reference coordinate system adopted in Figure 6, passing through the junctions that connect it to the supports; if this rotation is such that the angle between the $y$-and the $y^{\prime}$-axis in the sketches of Figure $6 \mathrm{~b}$ is equal to $\theta_{f}$, the field radiated by the field impinges on the TA surface with an angle that is actually $\theta_{f}$; in other words, it is sufficient to make a rotation of the coordinate system to overlap the drawing in Figure $6 a$ to that in Figure $6 \mathrm{~b}$ and this proves that they are equivalent. In the anechoic chamber, the measurement of the radiation pattern is done performing a 3D scanning in $(\theta, \phi)$, where $\theta$ and $\phi$ are the angles used in the spherical coordinate system; when the measurement in the E-plane (i.e., the $y z$-plane in Figure 6) is performed, the radiation pattern as a function of $\theta$ is obtained. Therefore, $\theta_{b}$ is immediately found in the correspondence of the direction of maximum radiation and operating a translation proportional to the rotation applied to the Transmitarray, i.e., the angle $\theta_{f}$.

The measured value of the main parameters characterizing the antenna radiation performances for the different $\theta_{f}$ are summarized in Table 1 . In the first column, the value of these quantities for broadside radiation, where the gain is maximum, are listed. In the considered range for $\theta_{f}$, the gain decreases less than $3 \mathrm{~dB}$, from a maximum of $29.97 \mathrm{~dB}$ at $0^{\circ}$ to $27.07 \mathrm{~dB}$ for $\theta_{f}= \pm 30^{\circ}$. This corresponds to an enlargement of the main beam, that is, however, never larger than $1^{\circ}$ in both planes (see 4 th and 5 th rows of the table). The decrease of the gain also affects the aperture efficiency, which reduces to $18 \%$ at the extremes of the scanning coverage. For what concerns the Side Lobe Levels (SLLs), their increase is more noticeable in the E-plane, where the beam is squinted, as also appears from the measured and simulated co-polarized radiation patterns at the frequency $f_{0}$ plotted in Figure 8 , for several values of the angle $\theta_{f}$. These patterns confirm the good scanning capabilities of the designed antenna, since they 
stay almost unchanged for scanning angles between $-20^{\circ}$ and $20^{\circ}$, but also, when $\theta_{f}=30^{\circ}$, they do not degrade significantly. Note that the maximum of the different plotted patterns occurs for angles $\theta_{b}$ that are slightly different from the corresponding angles $\theta_{f}$. In fact, even if theoretically $\theta_{b}=\theta_{f}$, in practice, this condition is not verified especially when these angles increase and what happens is that $\theta_{f}$ is typically less than $\theta_{b}$ [28]. The ratio between the main scanning beam direction and the feed offset angle can be defined as the Beam Deviation Factor (BDF), i.e., $\mathrm{BDF}=\theta_{b} / \theta_{f}$. For a very large scanning angle range, this parameter has to be optimized to obtain the desired performances. In the case of the designed TA, the values of the BDF are reported in the 7th row of Table 1: since it is equal to 0.9 , it means that the achieved range of variation for $\theta_{b}$ is of $54^{\circ}$.

Table 1. Transmitarray performances for different scanning angles.

\begin{tabular}{ccccc}
\hline $\boldsymbol{\theta}_{\boldsymbol{f}}$ & $\mathbf{0}^{\circ}$ & $\mathbf{\pm 1 0 ^ { \circ }}$ & $\mathbf{\pm 2 0 ^ { \circ }}$ & $\mathbf{\pm 3 0 ^ { \circ }}$ \\
\hline Gain & $29.97 \mathrm{dBi}$ & $29.30 \mathrm{dBi}$ & $27.96 \mathrm{dBi}$ & $27.07 \mathrm{dBi}$ \\
Ap. Eff. (30 GHz) & $35.1 \%$ & $30.1 \%$ & $22.2 \%$ & $18.1 \%$ \\
HPBW $_{\boldsymbol{E}}$ (30 GHz) & $3.8^{\circ}$ & $3.9^{\circ}$ & $4.1^{\circ}$ & $4.3^{\circ}$ \\
HPBW $_{\boldsymbol{H}}$ (30 GHz) & $3.9^{\circ}$ & $3.9^{\circ}$ & $4.1^{\circ}$ & $4.8^{\circ}$ \\
SLL $_{E}$ (30 GHz) & $-22.6 \mathrm{~dB}$ & $-20.4 \mathrm{~dB}$ & $-16.0 \mathrm{~dB}$ & $-11.1 \mathrm{~dB}$ \\
SLL $_{\boldsymbol{H}}$ (30 GHz) & $-22.6 \mathrm{~dB}$ & $-22.4 \mathrm{~dB}$ & $-20.1 \mathrm{~dB}$ & $-15.5 \mathrm{~dB}$ \\
BDF & 1 & 0.9 & 0.9 & 0.9 \\
X-pol (30 GHz) & $-40.4 \mathrm{~dB}$ & $-39.6 \mathrm{~dB}$ & $-38.5 \mathrm{~dB}$ & $-36.3 \mathrm{~dB}$ \\
1-dB BW & $23.5 \%$ & $24.5 \%$ & $23.3 \%$ & $24 \%$ \\
\hline
\end{tabular}

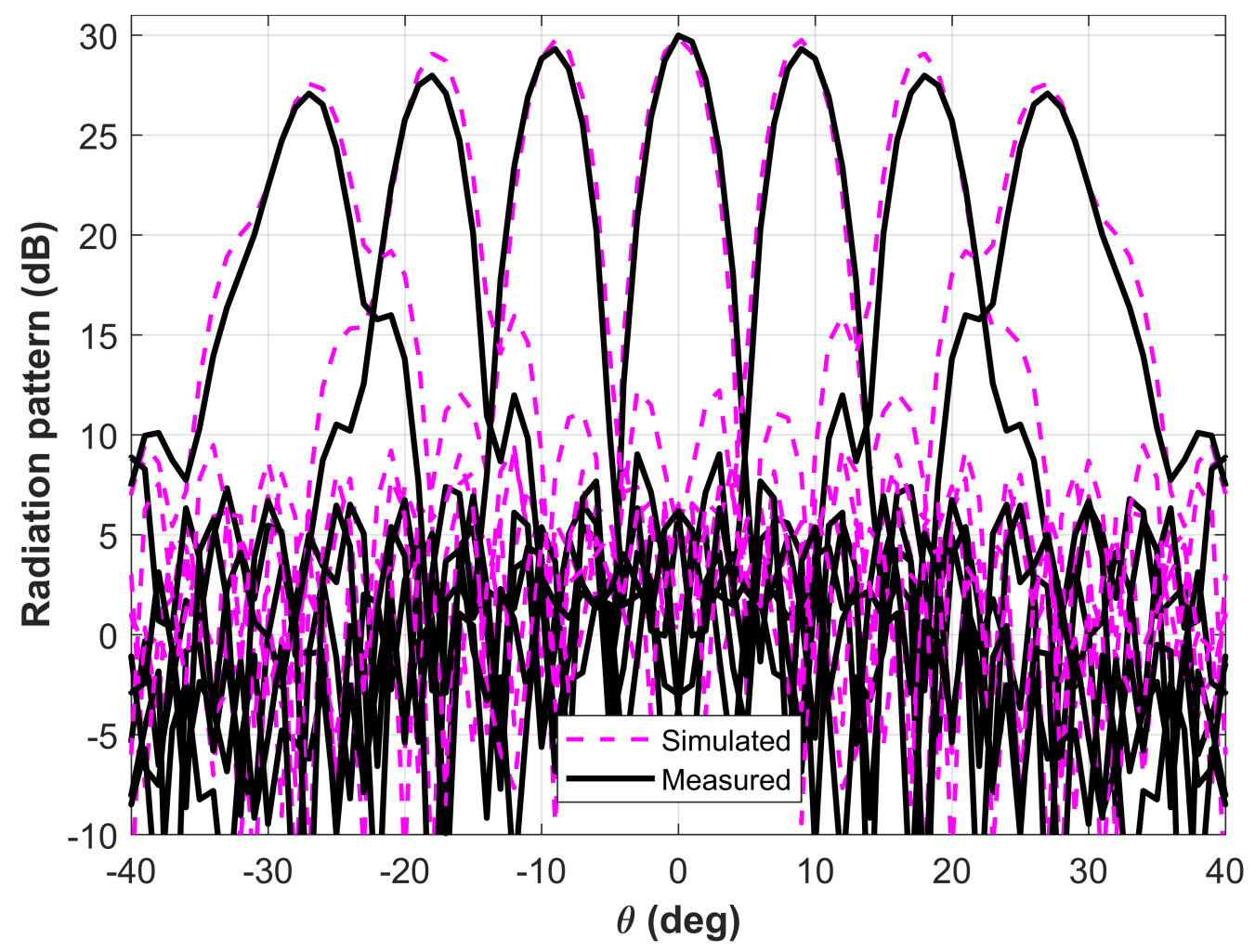

Figure 8. Simulated and measured co-polar radiation pattern in the E-plane at $30 \mathrm{GHz}$ for different scanning angles. 


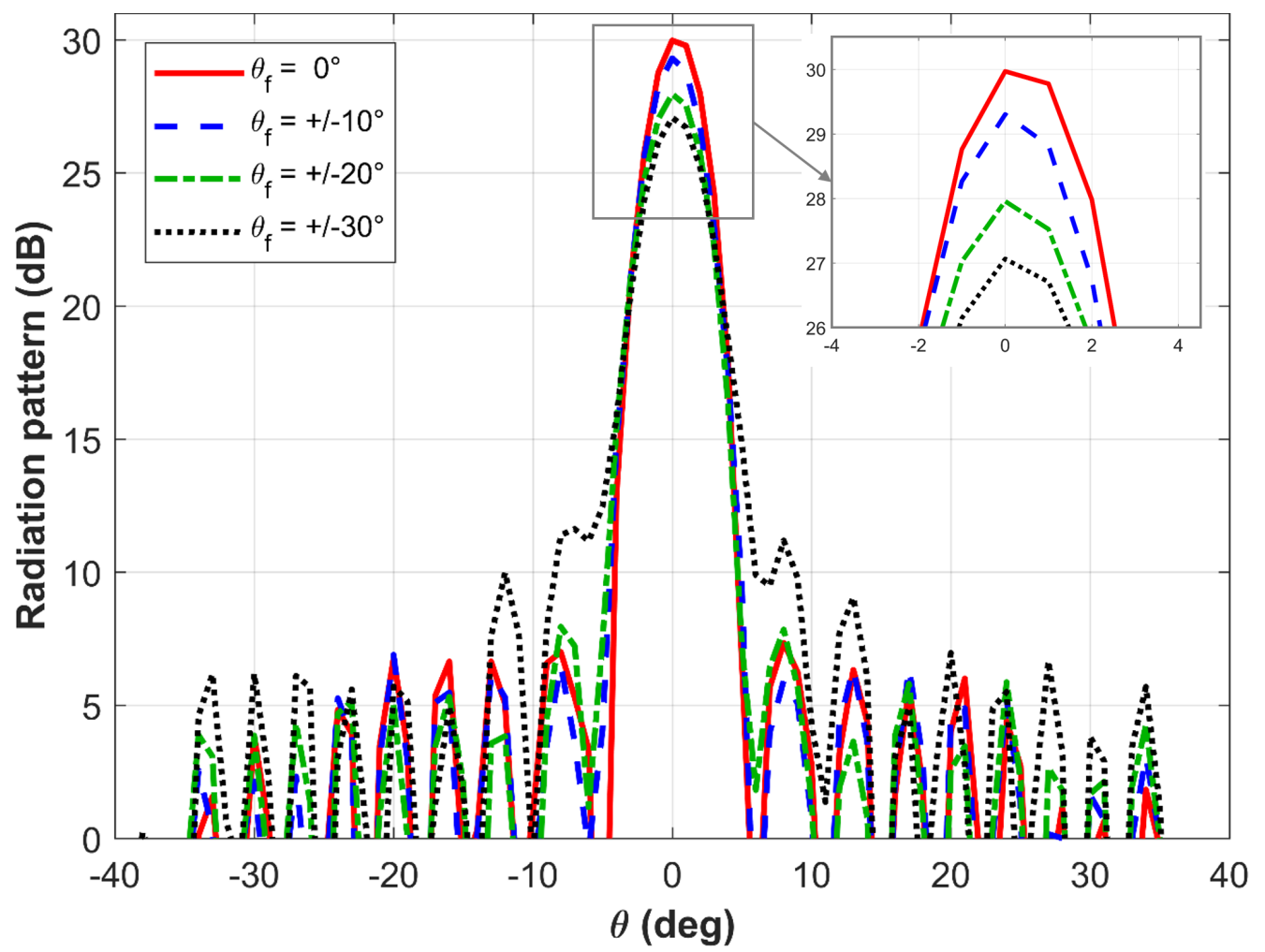

Figure 9. Measured co-polar radiation pattern in the $\mathrm{H}$-plane at $30 \mathrm{GHz}$ for different scanning angles.

Figure 9 shows the measured radiation patterns in H-plane, with a blow-up of the region close to the maximum in the inset. Apart from the reduction of the maximum gain, the radiation patterns are almost unchanged for $-20^{\circ} \leq \theta_{f} \leq 20^{\circ}$, and also for $\theta_{f}= \pm 30^{\circ}$ the most significant effect is just a contained increase of the side lobes. In both Figures 8 and 9, only the co-polar component of the radiated field is shown, to not make the plot incomprehensible, but the values of the cross-pol measured at $30 \mathrm{GHz}$ for each considered pointing angle are reported in Table 1: in all cases, it stays very low, reaching a maximum value of about $-36 \mathrm{~dB}$.

In Figure 10, the frequency variation of the gain for the different scanning angles is plotted, while the values of the $1-\mathrm{dB}$ bandwidth are reported in the last row of Table 1 . For the different values of $\theta_{f}$, the $1-\mathrm{dB}$ bandwidth remains larger than $23 \%$ and this represents an advantageous feature with respect to other transmitarray configurations implemented with metallic-dielectric elements, which have in most cases a narrow band and it decreases furthermore when the direction of maximum radiation changes. In Figure 11, the behavior of the measured and simulated gain at the design frequency as a function of the scanning angle is finally shown: the flatness of the curves is a further proof of the excellent scanning capabilities of the designed antenna; moreover, it is worth noticing the good agreement between the simulated and measured results. 


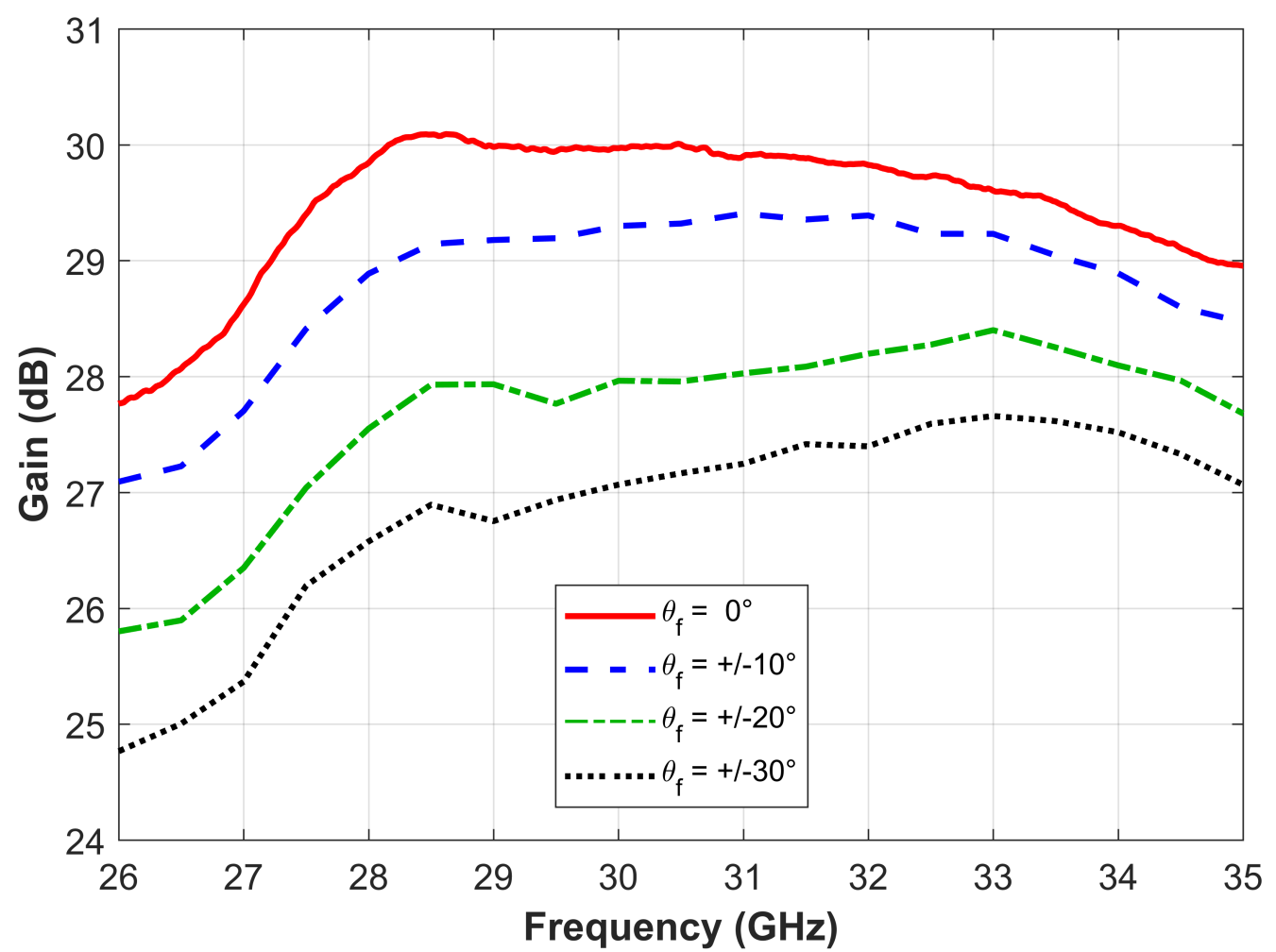

Figure 10. Measured frequency behaviour of the gain for different scanning angles.

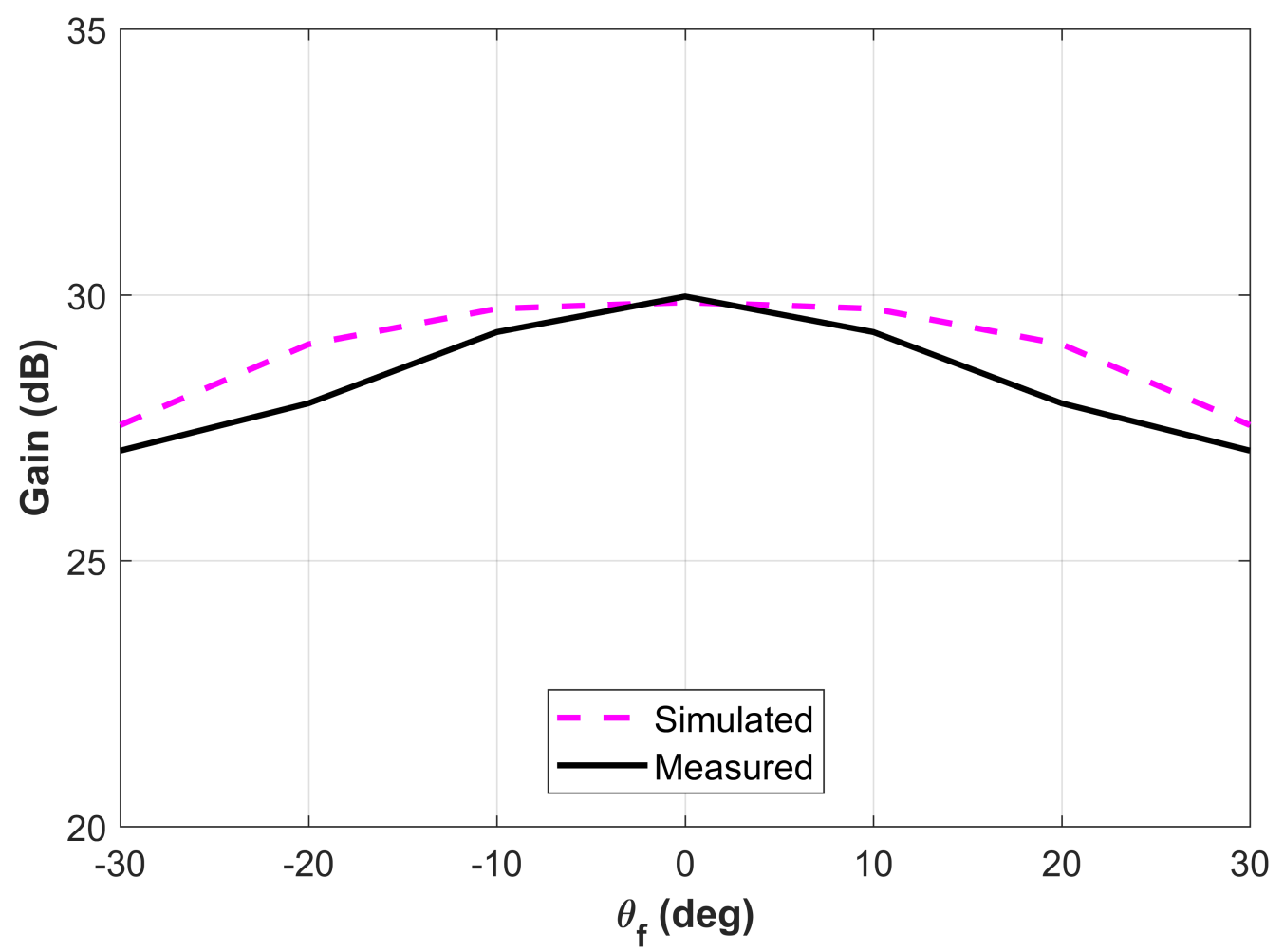

Figure 11. Comparison between the simulated and measured variation of the gain as a function of $\theta_{f}$. 


\section{Conclusions}

In this paper, the beam scanning capabilities of a 3D-printed dielectric transmitarray antenna have been analyzed and experimentally validated. The radiation patterns measured for different scanning angles in the range $\pm 27^{\circ}$ showed a small variation of the gain, a controlled increase of the SLL and a wideband behavior, confirming the expected antenna properties. While these results prove the suitability of the considered unit-cell for the design of multibeam or beam scanning passive transmitarrays, some further optimization of the antenna would be necessary if similar performances would be obtained on larger scanning coverage. It will be the object of future investigations.

Author Contributions: Conceptualization, A.M. and P.P.; methodology, A.M. and P.P; validation, G.D.; formal analysis, A.M.; investigation, A.M.; resources, P.P. and G.D.; data curation, A.M.; writing-original draft preparation, A.M. and P.P.; writing-review and editing, P.P.

Funding: This work was supported by the General Directorate for Cultural and Economic Promotion and Innovation of the Ministry of Foreign Affairs and International Cooperation, of the Italian Republic.

Acknowledgments: The authors thank the research group of Chilab for the antenna manufacturing.

Conflicts of Interest: The authors declare no conflict of interest.

\section{References}

1. Huang, J.; Encinar, J.A. Reflectarray Antennas; Wiley IEEE Press: Hoboken, NJ, USA, 2008.

2. Abdelrahman, A.H.; Yang, F.; Esherbeni, A.Z.; Nayeri, P. Analysis and Design of Transmitarray Antennas; M\&C Publishers: San Francisco, CA, USA, 2017.

3. Ryan, C.G.M.; Chaharmir, M.R.; Shaker, J.R.B.J.; Bray, J.R.; Antar, Y.M.; Ittipiboon, A. A wideband transmitarray using dual-resonant double square rings. IEEE Trans. Antennas Propag. 2010, 58, 1486-1493. [CrossRef]

4. Abdelrahman, A.H.; Elsherbeni, A.Z.; Yang, F. High-gain and broadband transmitarray antenna using triple-layer spiral dipole elements. IEEE Antennas Wirel. Propag. Lett. 2014, 13, 1288-1291. [CrossRef]

5. Cai, Y.M.; Li, W.; Li, K.; Gao, S.; Yin, Y.; Zhao, L.; Hu, W. A Novel Ultra-Wideband Transmitarray Design Using Tightly Coupled Dipole Elements. IEEE Trans. Antennas Propag. 2019, 67, 242-250. [CrossRef]

6. Abdelrahman, A.H.; Esherbeni, A.Z.; Yang, F. Transmitarray antenna design using cross slot elements with no dielectric substrate. IEEE Antennas Wirel. Propag. Lett. 2014, 13, 177-180. [CrossRef]

7. Rahmati, B.; Hassani, H.R. High-efficient wideband slot transmitarray antenna. IEEE Trans. Antennas Propag. 2015, 63, 5149-5155. [CrossRef]

8. Mahmoud, A.-E.; Hong, W.; Zhang, Y.; Kishk, A. W-band mutlilayer perforated dielectric substrate lens. IEEE Antennas Wirel. Propag. Lett. 2014, 13, 734-737. [CrossRef]

9. Yi, H.; Qu, S.W.; Ng, K.B.; Chan, C.H.; Bai, X. 3-D printed millimeter-wave and terahertz lenses with fixed and frequency scanned beam. IEEE Trans. Antennas Propag. 2016, 64, 442-449. [CrossRef]

10. Massaccesi, A.; Pirinoli, P. Enhancing the bandwidth in transmitarray antennas using tapered transmission line matching approach. In Proceedings of the 12th European Conference on Antennas and Propagation (EuCAP 2018), London, UK, 9-13 April 2018.

11. Massaccesi, A.; Pirinoli, P.; Vardaxoglou, J.C. A multilayer unit-cell for perforated dielectric transmitarray antennas. In Proceedings of the 2018 IEEE International Symposium on Antennas and Prop \& USNC/URSI National Radio Science Meeting, Boston, MA, USA, 8-13 July 2018; pp. 263-264.

12. Massaccesi, A.; Pirinoli, P.; Bertana, V.; Scordo, G.; Marasso, S.L.; Cocuzza, M.; Dassano, G. 3D-Printable dielectric transmitarray with enhanced bandiwidth at millimiter-waves. IEEE Access 2018, 6, 46407-46418. [CrossRef]

13. Beccaria, M.; Massaccesi, A.; Pirinoli, P. Multibeam transmitarrays for 5G antenna systems. In Proceedings of the 2018 IEEE Seventh International Conference on Communications and Electronics (ICCE), Hue, Vietnam, 18-20 July 2018; pp. 217-221.

14. Jiang, M.; Chen, Z.N.; Zhang, Y.; Hong, W.; Xuan, X. Metamaterial-based thin planar lens antenna for spatial beamforming and multibeam massive MIMO. IEEE Trans. Antennas Propag. 2017, 65, 464-472. [CrossRef] 
15. Hou, Y.; Chang, L.; Li, Y.; Zhang, Z.; Feng, Z. Linear Multibeam Transmitarray Based on the Sliding Aperture Technique. IEEE Trans. Antennas Propag. 2018, 66, 3948-3958. [CrossRef]

16. Liu, G.; Pham, K.; Cruz, E.M.; Ovejero, D.G.; Sauleau, R. A millimeter wave transparent transmitarray antenna using meshed double circle rings elements. In Proceedings of the 12th European Conference on Antennas and Propagation (EuCAP 2018), London, UK, 9-13 April 2018.

17. Lima, E.B.; Matos, S.A.; Costa, J.R.; Fernandes, C.A.; Fonseca, N.J.G. Circular polarization wide-angle beam steering at Ka-band by in-plane translation of a plate lens antenna. IEEE Trans. Antennas Propag. 2015, 63, 5443-5455. [CrossRef]

18. Liu, G.; Cruz, E.M.; Pham, K.; Ovejero, D.G.; Sauleau, R. Low Scan Loss Bifocal Ka-band Transparent Transmitarray Antenna. In Proceedings of the 2018 IEEE International Symposium on Antennas and Prop \& USNC/URSI National Radio Science Meeting, Boston, MA, USA, 8-13 July 2018; pp. 1449-1450.

19. Clemente, A.; Dussopt, L.; Sauleau, R.; Potier, P.; Pouliguen, P. Wideband 400-Element Electronically Reconfigurable Transmitarray in X Band. IEEE Trans. Antennas Propag. 2013, 61, 5017-5027. [CrossRef]

20. Pan, W.; Huang, C.; Ma, X.; Jiang, B.; Luo, X. A dual linearly polarized transmitarray element with 1-Bit phase resolution in X-band. IEEE Antennas Wirel. Propag. Lett. 2015, 14, 167-171. [CrossRef]

21. Di Palma, L., Clemente, A., Dussopt, L., Sauleau, R., Potier, P., Pouliguen, P. Circularly-polarized reconfigurable transmitarray in Ka-band with beam scanning and polarization switching capabilities. IEEE Trans. Antennas Propag. 2017, 65, 529-540. [CrossRef]

22. Nguyen, D.B.; Pichot, C. Unit-Cell loaded with PIN diodes for 1-bit linearly polarized reconfigurable transmitarrays. IEEE Antennas Wirel. Propag. Lett. 2019, 18, 98-102. [CrossRef]

23. Padilla, P.; Munoz-Acevedo, A.; Sierra-Castaner, M.; Sierra-Perez, M. Electronically reconfigurable transmitarray at Ku band for microwave applications. IEEE Trans. Antennas Propag. 2010, 58, 2571-2579. [CrossRef]

24. Lau, J.Y.; Hum, S.V. A wideband reconfigurable transmitarray element. IEEE Trans. Antennas Propag. 2012, 60, 1303-1311. [CrossRef]

25. Lau, J.Y.; Hum, S.V. Reconfigurable transmitarray design approaches for beamforming applications. IEEE Trans. Antennas Propag. 2012, 60, 5679-5689. [CrossRef]

26. Sazegar, M.; Yuliang, Z.; Kohler, C.; Maune, H.; Nikfalazar, M.; Binder, J.R.; Jakoby, R. Beamsteering transmitarray using tunable frequency selective surface with integrated ferroelectric varactors. IEEE Trans. Antennas Propag. 2012, 60, 5690-5699. [CrossRef]

27. Huang, C.; Pan, W.; Ma, X.; Jiang, B.; Cui, J.; Luo, X. Using reconfigurable transmitarray to achieve beam-steering and polarization manipulation applications. IEEE Trans. Antennas Propag. 2015, 63, 4801-4810. [CrossRef]

28. Rengarajan, S.R. Scanning and defocusing characteristics of microstrip reflectarray. IEEE Antennas Wirel. Propag. Lett. 2010, 9, 163-166. [CrossRef]

(C) 2019 by the authors. Licensee MDPI, Basel, Switzerland. This article is an open access article distributed under the terms and conditions of the Creative Commons Attribution (CC BY) license (http:/ / creativecommons.org/licenses/by/4.0/). 